\title{
Small Spheres Freeze When Hot
}

\author{
An optofluidic effect causes a group of fluid-suspended particles to \\ "freeze" when one of them is heated, potentially allowing greater control \\ over these systems.
}

\section{By Marric Stephens}

A material that freezes when heated and melts when cooled defies intuition. But such a system has been demonstrated by Alessio Caciagli and colleagues at the University of Cambridge in the UK [1]. Suspending particles in a fluid and heating just one of them, the team show that they can initiate a particle-attracting flow that aggregates particles and "freezes" them into a two-dimensional crystal. The result shows how particles that are not themselves optically trapped might still be manipulated with lasers.

Moving particles in fluids with temperature gradients is a well-studied problem. Typically, suspended particles move from hot to cold regions, while fluid molecules move from cold to hot. In their experiment, Caciagli and colleagues engineered the opposite situation.

The experiment took place at the upper surface of an oil drop sitting in a bath of water. The team attached micrometer-sized polystyrene particles to the water-oil interface via DNA "tethers," such that the particles could move through the water
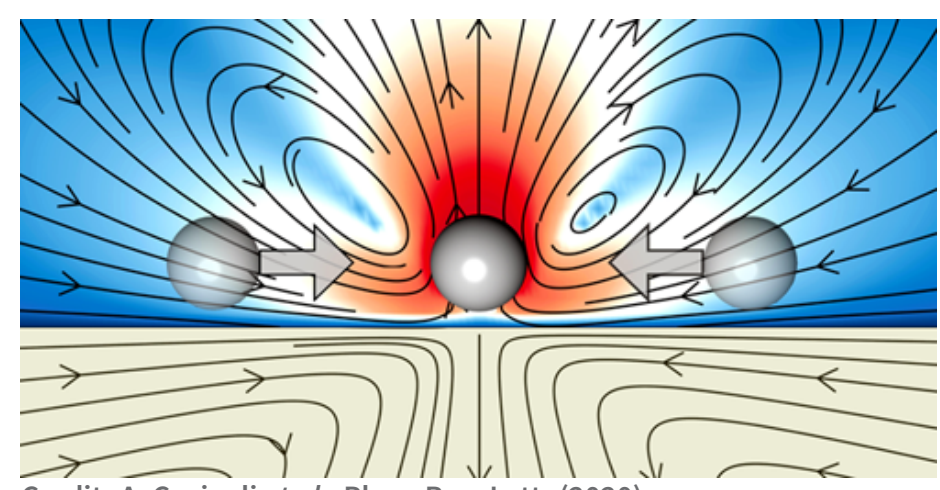

Credit: A. Caciagli et al., Phys. Rev. Lett. (2020)

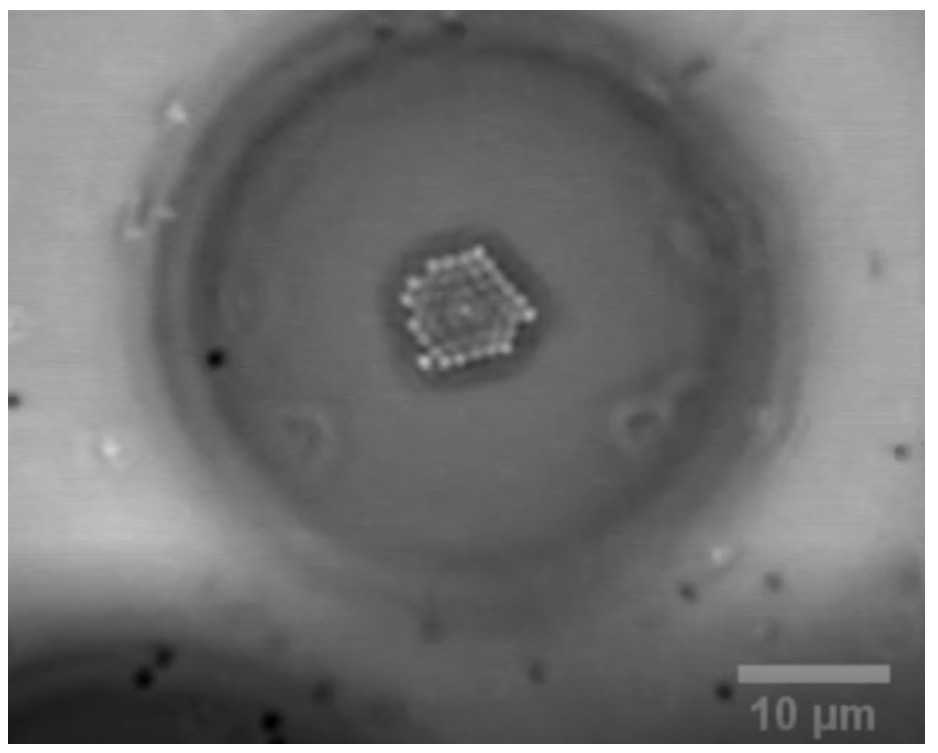

Video 1: Particles form a two-dimensional crystal under the influence of temperature-induced fluid flow.

Credit: A. Caciagli et al., Phys. Rev. Lett. (2020)

around the drop but could not enter the oil. They then trapped one particle using a laser. The laser warmed the particle by $5^{\circ} \mathrm{C}$ and created a temperature gradient in the surrounding water. Constrained by the DNA tether, the particle then moved toward the oil, triggering a flow along the fluid interface. This fluid flow sucked in neighboring tethered particles, which packed together into a crystal.

The researchers say that switching the thermal conductivities of the two fluids would reverse the flow's direction. Such optofluidic control could lead to controlled self-assembly of fluid-suspended particles and might offer new ways to fabricate metamaterials. 
This research is published in Physical Review Letters.

Marric Stephens is a Corresponding Editor for Physics based in Bristol, UK.

\section{REFERENCES}

1. A. Caciagli et al., "Controlled optofluidic crystallization of colloids tethered at interfaces," Phys. Rev. Lett. 125, 068001 (2020). 